\title{
Time-Dependent Effects of Repeated Amphetamine Treatment on Norepinephrine in the Hypothalamus and Hippocampus Assessed with In Vivo Microdialysis
}

\author{
Dianne M. Camp, Ph.D., Donna K. DeJonghe, and Terry E. Robinson, Ph.D.
}

The effects of repeated amphetamine $(A M P H)$ pretreatment on norepinephrine (NE) neurotransmission in the hypothalamus and hippocampus were assessed using in vivo microdialysis. Rats were pretreated with either saline or an escalating-dose AMPH regimen $(1 \rightarrow 10 \mathrm{mg} / \mathrm{kg})$ over 10 consecutive days, and then were withdrawn from AMPH for either 1 day or 30 days, at which time the animals underwent two consecutive days of testing. As expected, repeated treatment with AMPH resulted in timedependent changes in both spontaneous locomotor activity and in the psychomotor response to a subsequent challenge injection of AMPH. In addition, repeated exposure to AMPH resulted in time-dependent and regionally-specific changes in the basal concentrations of NE in dialysate, and in the NE response to an AMPH challenge. For example, $A M P H$ pretreatment produced a persistent (at least one month) increase in the basal concentration of NE in the hippocampus, but not the hypothalamus, although the response to an $A M P H$ challenge was altered in both structures. It is suggested that AMPH treatment produces adaptations in NE systems that far outlast the acute effects of the drug, and that these may contribute to both transient and more persistent behavioral sequelae associated with the discontinuation of chronic AMPH use.

[Neuropsychopharmacology 17:130-140, 1997] (C) 1997 American College of Neuropsychopharmacology. Published by Elsevier Science Inc.
KEY WORDS: Hippocampus; Hypothalamus; Amphetamine; Behavioral depression; Behavioral sensitization; Drug withdrawal; Norepinephrine; Microdialysis; Locomotor activity

There are a number of time-related alterations in brain and behavior in both human and non-human animals as a consequence of repeated exposure to psychostimulant drugs, such as amphetamine (AMPH). In humans, the discontinuation of chronic AMPH use initially re-

From the Biopsychology and Neuroscience Programs, Department of Psychology, The University of Michigan, 525 East University Street, Ann Arbor, MI 48109-1109.

Address correspondence to: Dianne M. Camp, Ph.D., Clinical Neuroscience Laboratory, Research Building, Room 209, Sinai Hospital, 6767 West Outer Drive, Detroit, MI 48235.

Received May 23, 1996; accepted March 4, 1997. sults in a withdrawal syndrome that is reported to include mood changes that resemble some symptoms of depression (Gawin and Ellinwood 1988; Jaffe 1990; Watson et al. 1972). In rats, a post-AMPH withdrawal 'depression' in behavior has been described, and its major features include a decrease in nocturnal locomotor activity, a decrease in the efficacy of intracranial selfstimulation reward, and an insensitivity to external stimulation (Cassens et al. 1981; Herman et al. 1971; Kokkinidis and Zacharko 1980; Leith and Barrett 1976; Paulson et al. 1991; Robinson and Camp, 1987; Schreiber et al. 1976; Tonge 1974). These effects are relatively transient, usually lasting for only 1 to 10 days after the discontinuation of AMPH treatment (Cassens et al. 1981; Leith and Barrett 1976; Paulson et al. 1991). Repeated $\mathrm{AMPH}$ treatment also produces, however, very long- 
lasting adaptations characterized by the phenomenon of sensitization. For example, animals pretreated with AMPH are hypersensitive (sensitized) to the psychomotor stimulant (Robinson and Becker 1986; Stewart and Badiani 1993) and rewarding effects (Lett 1989; Piazza et al. 1989) of AMPH, and this hypersensitivity to the drug can persist for up to 1 year following discontinuation of AMPH treatment (Paulson et al. 1991; Valadez and Schenk 1994). Similarly, former AMPH addicts are reported to show a persistent hypersensitivity to the psychotomimetic effects of AMPH (Sato 1983).

Most studies addressing the neurobiological basis of the AMPH withdrawal syndrome and AMPH sensitization have concentrated on brain dopamine (DA) systems, and a number of time-dependent adaptations have been identified in mesotelencephalic dopamine neurotransmission (Kokkinidis 1988; Paulson and Robinson 1995, 1996; Rosetti et al. 1992; Kalivas and Stewart 1991; Robinson and Becker 1986; Stewart and Badiani 1993; White and Wolf 1991). In addition to its effects on dopamine neurotransmission, however, AMPH has potent effects on norepinephrine (NE) systems (Kuczenski and Segal 1992), and it is possible that alterations in NE neurotransmission also may contribute to the timedependent changes in behavior produced by chronic AMPH administration. Thus, the purpose of this study was to characterize the effects of repeated exposure to AMPH on NE neurotransmission both early (1-2 days) and late (30-31 days) following the discontinuation of treatment with escalating doses of AMPH. This was accomplished using in vivo microdialysis in freely moving rats to sample NE in both the hippocampus and hypothalamus. These two brain regions were selected for study because they represent the two major projection targets for the locus coeruleus and lateral tegmental NE systems, respectively (Lindvall and Bjorklund 1983; Moore and Bloom 1979).

\section{METHODS}

\section{Subjects}

Male Holtzman rats weighing 200-225 g at the beginning of the experiment were housed individually in wire hanging cages in a light (14/10 light/dark cycle, lights on at $06: 00 \mathrm{hr})$ and temperature $\left(68-74^{\circ} \mathrm{F}\right)$ controlled room. Food and water were provided ad lib.

\section{Amphetamine Pretreatment Regimen and Groups}

Animals were pretreated with $d$-amphetamine sulfate (AMPH; weight of the salt) using an escalating-dose regimen (1 to $10 \mathrm{mg} / \mathrm{kg}$, intraperitoneal (i.p.)), in which the drug was administered twice daily (approximately 8 hours apart), for ten consecutive days, and each day the dose was incremented by $1 \mathrm{mg} / \mathrm{kg}$. Control rats re- ceived $1 \mathrm{ml} / \mathrm{kg}$ of $0.9 \%$ saline. All injections, except the last one, were given in the home cage. Each animal was assigned to one of four pretreatment-test groups: Group 1 underwent dialysis testing both 1 and 2 days following discontinuation of pretreatment with AMPH; Group 2 was tested after 30 and 31 days of AMPH withdrawal; Groups 3 and 4 were saline-pretreated controls tested either 1-2 days or 30-31 days after the last injection of saline, respectively. Furthermore, half of the animals in each group received a microdialysis probe in the hypothalamus, and half received a probe in the dorsal hippocampus. Thus, there were a total of eight independent groups of animals. Finally, approximately equal numbers of AMPH-pretreated and saline control animals were tested at the same time, but animals with a probe in the hippocampus were tested separately from animals with a probe in the hypothalamus.

\section{Procedures}

Rats were anesthetized with sodium pentobarbital (supplemented with methoxyflurane, if necessary), and then, using standard stereotaxic surgical procedures, all animals had a 21 gauge stainless steel guide cannula placed on the dural surface above either the hippocampus or hypothalamus. In addition, a length of 15 gauge stainless steel tubing was located behind the guide cannula to be later used as an anchor for a tether. The entire assembly was fixed in place using jeweler's screws and cranioplastic cement. The coordinates were: (for paraventricular nucleus of the hypothalamus) posterior to bregma, $1.6 \mathrm{~mm}$; lateral, $0.6 \mathrm{~mm}$; and ventral, $1.0 \mathrm{~mm}$ from the skull surface; (for the dorsal hippocampus) posterior, $3.8 \mathrm{~mm}$; lateral, $1.9 \mathrm{~mm}$; and ventral, $1.0 \mathrm{~mm}$ (Paxinos and Watson 1986). Surgery was performed 2 to 3 weeks before the microdialysis experiment, which meant that the animals tested after 1-2 days of drug withdrawal had surgery 4 to 10 days prior to the start of pretreatment injections; and animals tested after 30-31 days of withdrawal had surgery 9 to 15 days following the pretreatment injections.

On the day before the first dialysis session, each animal was placed into a $46 \times 27 \times 28.5 \mathrm{~cm}$ oval-shaped Plexiglas chamber. At this time, the 1-2 day withdrawal groups received their last injection of AMPH or saline. Then, during the afternoon on the same day, the animal was manually restrained, and a dialysis probe was quickly lowered via the guide cannula. The concentricshaped probe was basically as described by Robinson and Camp (1991), with one major difference. The stainless steel cannula part of the probe stopped at the bottom of the guide cannula with the rest of the probe length consisting of dialysis membrane. The dialysis membrane was coated with cyanoacrylate glue, except for the ventral $2.5 \mathrm{~mm}$ (for hypothalamus probes) or 2.0 $\mathrm{mm}$ (for hippocampus probes) tip. After lowering the 
probe, the animal was returned to the test cage and attached to a liquid swivel via a flexible steel tether made from model airplane control cable, which was fastened to the $15 \mathrm{~g}$ tubing fixed to the skull 'cap'. The perfusion medium, consisting of $145 \mathrm{mM} \mathrm{NaCl}, 2.7 \mathrm{mM} \mathrm{KCl}$, and

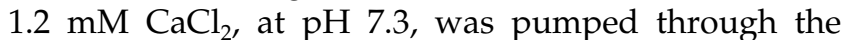
probe overnight at a rate of $0.3 \mu \mathrm{l} / \mathrm{min}$, during which time the animal was left undisturbed.

The following morning the pump was turned up to a flow rate of $1.5 \mu \mathrm{l} / \mathrm{min}$, and after about 1 hour, nine 30 minute baseline samples were collected. After this, the pumps were again turned down to $0.3 \mu \mathrm{l} / \mathrm{min}$, and the animal was left undisturbed in the test chamber overnight. On the next morning the above procedure was repeated, except only four 30 minute baseline samples were collected. After the last baseline sample was collected, each animal received an i.p. injection of $2.0 \mathrm{mg} /$ $\mathrm{kg}$ of $\mathrm{AMPH}$ and six additional 30 minute samples were collected.

\section{Behavior}

Locomotor activity was recorded in the test chambers via two photocells placed $23.5 \mathrm{~cm}$ apart and $4.5 \mathrm{~cm}$ above the cage floor. A single count was registered when the animal crossed the photocell beam, and another count from that photocell could not be registered until the other photocell beam was broken. Thus, movement from one side of the cage to the other (crossovers) was detected using this arrangement.

Locomotor activity (crossovers) was recorded on three separate occasions over the course of the dialysis experiment. Overnight activity was recorded during both the first and second nights after the probe was lowered, representing 10-24 hours and 34-48 hours following the discontinuation of AMPH treatment in the '1-2 day' treatment groups. Locomotor activity was also recorded 30 minutes prior to and for 3 hours following the AMPH challenge injection given during the second day of dialysate collection. In addition, a single visual rating of drug-induced behavior was taken at 30 minutes following the AMPH challenge using the following 4-point scale: 1 = normal, alert, active; $2=$ hyperactive with or without intermittent stereotypy; $3=$ continuous stereotypy over a wide area; and $4=$ continuous stereotypy over a restricted area.

\section{Assay of Dialysate}

Dialysate was collected into tubes containing $5 \mu \mathrm{l}$ of mobile phase (described below), and was immediately frozen on dry ice and stored at $-70^{\circ} \mathrm{C}$ until assayed. Dialysate samples were assayed for NE by HPLC with electrochemical detection. NE was separated on a $150 \times$ $3 \mathrm{~mm}$ BDS-Hypersil-C18 column (Keystone Scientific) maintained at a constant temperature of $40-42^{\circ} \mathrm{C}$. The mobile phase consisted of $75 \mathrm{mM}$ sodium phosphate, $0.50 \mathrm{mM}$ sodium dodecyl sulfate, $25 \mu \mathrm{M}$ (ethylenedinitrile)-tetraacetic acid (EDTA), 10\% acetonitrile, 5\% methanol, and with a final $\mathrm{pH}$ of 5.6. Dialysate was injected manually onto the column using a Valco injection valve. Detection was accomplished using an ESA Coulochem II detector, ESA guard cell (set at an oxidizing potential of $300 \mathrm{mV}$ ) and ESA Model 5014 analytical cell (with electrode 1 set at a reducing potential of $-175 \mathrm{mV}$ and electrode 2 set at an oxidizing potential of $175 \mathrm{mV}$ ). Under these conditions, NE could be quantified at both electrodes, and thus, oxidation-reduction ratios could be calculated for each sample. The output signals from the detector were analyzed on-line using a computerbased data acquisition system (EZ Chrom, Scientific Software, Inc., San Ramon, CA).

The NE peak in dialysate was identified by comparison of the elution times of dialysate NE and authentic NE standards prepared in the perfusion medium also containing mobile phase. Blank samples consisting of the perfusion medium plus mobile phase were analyzed to determine if this produced interfering peaks. The peak was identified as NE in two additional ways. First, oxidation-reduction ratios were determined for the putative NE peak in dialysate and an authentic NE standard, and when compared these ratios were shown to be the same. Second, a subgroup of animals was given an i.p. injection of $0.3 \mathrm{mg} / \mathrm{kg}$ of clonidine, and as expected for NE, this eliminated the NE signal (Abercrombie and Finley 1991; L'Heureux et al. 1986).

\section{Histology}

At the end of the experiment the animals were given an overdose of sodium pentobarbital and perfused through the heart with $0.9 \%$ saline, followed by $10 \%$ formalinsaline solution. The brain was removed and stored in $10 \%$ formalin-saline solution, and a few days before sectioning was transferred to a $30 \%$ sucrose-formalin solution. The brain was sectioned using a frozen technique, was stained with cresyl violet and examined to determine the exact location of the dialysis probe.

\section{Data Analysis}

Locomotor activity (crossovers) and neurochemical data were analyzed using two-way analyses of variance with repeated measures for overall group comparisons, and if significant, were followed by subsequent analyses of variance and/or Fisher's PLSD post hoc tests for pairwise group comparisons using Statview statistical software (Abacus Concepts). Preliminary analyses were conducted to determine whether the data for the two saline control groups (i.e., 1-2 day and 30-31 day withdrawal groups) could be pooled. There were no differences in the behavior or neurochemistry for these groups, 
and therefore, all subsequent analyses performed were done on the pooled data for these groups.

In vitro recovery was performed on all dialysis probes prior to each use (probes were used one to three times), and dialysate values reported here were corrected for probe recovery. The average $( \pm$ SEM) recovery value for NE in vitro was $12.04 \pm 0.38 \%$ for the 2 $\mathrm{mm}$ probes and $15.70 \pm 0.67 \%$ for the $2.5 \mathrm{~mm}$ probes. The recovery values did not differ significantly between treatment groups.

\section{RESULTS}

\section{Dialysis Probe Placements}

Figure 1 shows the location of the dialysis surface with the probes located either in the hypothalamus or hippocampus for all animals for which neurochemical data were obtained. The majority of probe placements in the hypothalamus were centered in the medial hypothalamus at the level of the paraventricular nucleus. Hippocampal probes spanned the dorso-ventral extent of the dorsal hippocampus.

\section{Locomotor Behavior (Crossovers)}

There were no differences in crossovers across the daynight cycle between animals with probes in the hypothalamus versus the hippocampus. Therefore, these groups were pooled for analysis and presentation of the behavioral data. Figure 2 shows spontaneous locomotor activity (crossovers) during the first night after a dialysis probe was lowered. All groups showed a large peak in locomotor activity when the lights went out. This was followed by moderate levels of activity during the middle of the night and end of the lights-off period.
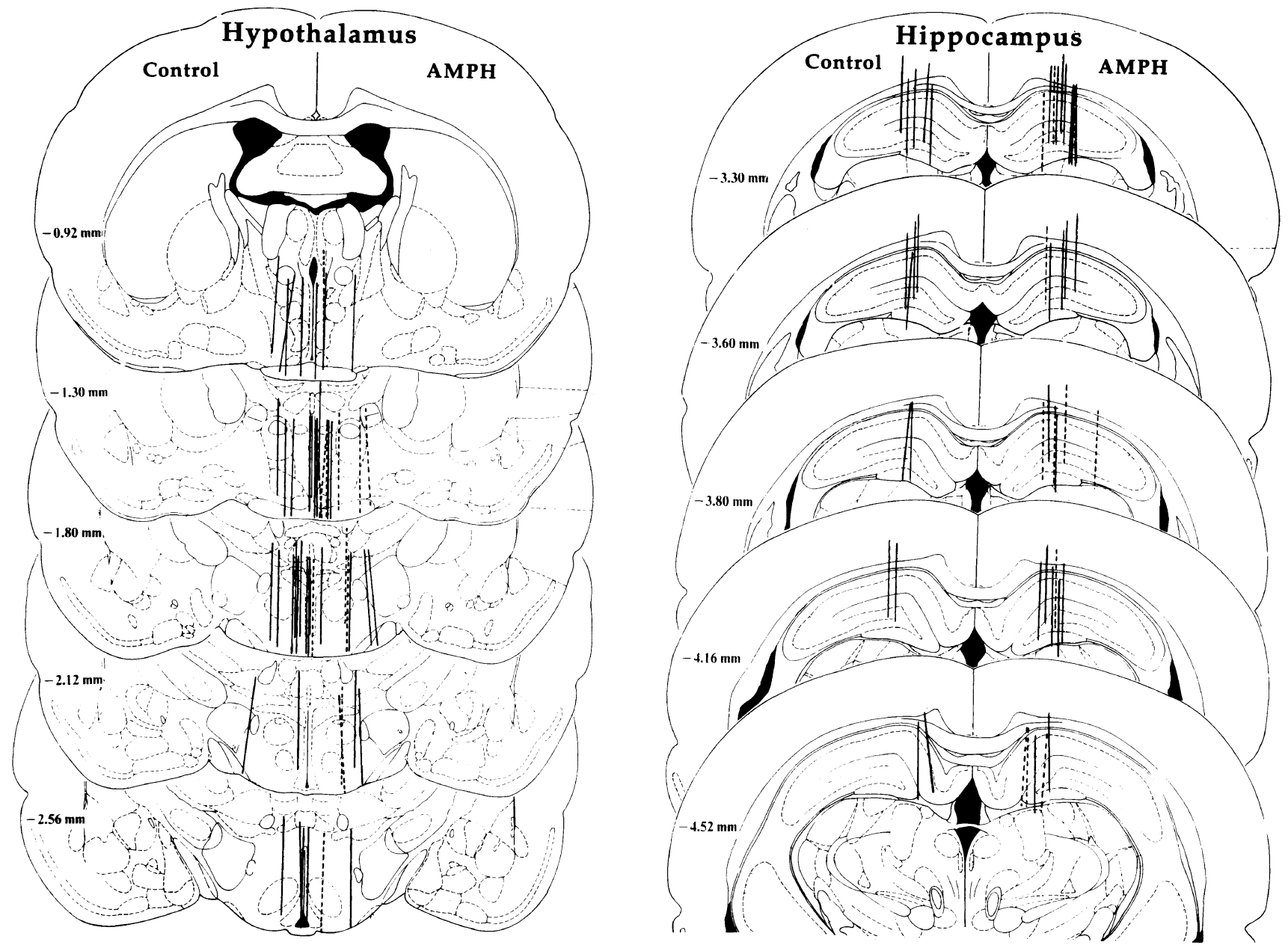

Figure 1. Schematic drawings of coronal sections of the rat brain adapted from the atlas of Paxinos and Watson (1986), showing the location of the dialysis surface of probes in either the hypothalamus or dorsal hippocampus. Probe locations in control (saline-pretreated) animals are shown on the left-hand side of each section and those in AMPH-pretreated animals on the right-hand side of each section. In addition, AMPH-pretreated animals withdrawn for 1-2 days are depicted by the solid lines and animals withdrawn for 30-31 days by the dashed lines. The number on each drawing indicates the distance posterior to bregma. 
AMPH-pretreated animals withdrawn from AMPH for 1 day were significantly less active during the middle of the night and the end of the lights-off period compared to controls and AMPH-pretreated animals withdrawn from AMPH for 30 days. This is consistent with earlier reports (Robinson and Camp 1987; Paulson et al. 1991). AMPH-pretreated animals withdrawn from AMPH for one day also showed nocturnal hypoactivity the following night as well (data not shown). However, there were no significant differences in locomotor activity between AMPH-pretreated and control animals during the lights-on period, when all animals were relatively inactive.

Figure 3 shows the effects of a challenge injection of $2.0 \mathrm{mg} / \mathrm{kg}$ of AMPH on locomotor activity in AMPHand saline-pretreated animals. AMPH produced a large increase in locomotor activity in all groups, but there were significant group differences in the pattern of activity. Control animals and animals given a challenge

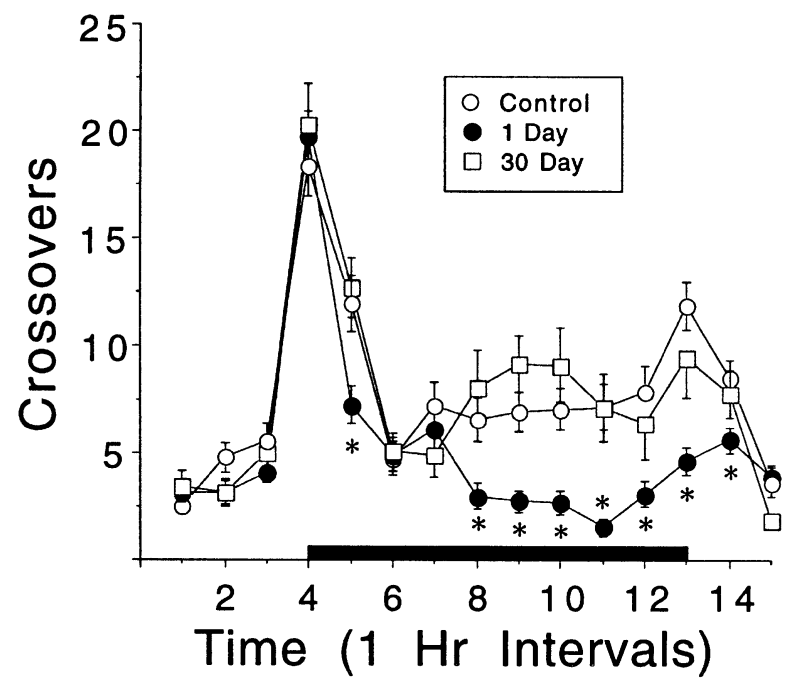

Figure 2. The mean number ( \pm S.E.M.) of crossovers per one hour interval during the 3 hours before the lights went off, the 10 hour lights-off period (as represented by the solid black bar), and the 2 hours after the lights came back on during the first night after lowering the probe in saline-pretreated Control animals, and animals withdrawn from AMPH for 10 to 24 hours (1 Day group) or 30 days (30 Day group).There were significant group differences in spontaneous locomotor activity, as indicated by an overall 2-way ANOVA (group effect $F=15.0, p<0.0001$; interaction effect $F=3.934, p<0.0001)$. Subsequent ANOVA's comparing two groups at a time showed that the 1 Day group differed from both the Control group (group effect $F=32.86, p<$ 0.0001 ; interaction effect $F=5.27, p<0.0001$ ) and the 30 Day group (group effect $F=17.67, p<0.0001$; interaction effect $F=5.48, p<0.0001)$. The Control and 30 Day groups did not differ from one another $(p>0.28)$. The asterisks indicate the time intervals when the 1 Day group differed from Control animals ( $p<0.05$, as determined by Fishers PLSD tests). Group $n$ values: Control $n=54 ; 1$ Day $n=64 ; 30$ Day $n=32$. injection of AMPH two days after AMPH withdrawal showed a large increase in locomotor activity that persisted for about 2 hours. Furthermore, peak locomotor activity was significantly greater in AMPH-pretreated animals withdrawn for 2 days than in saline-pretreated controls. In contrast, animals given an AMPH challenge 31 days after the discontinuation of AMPH pretreatment showed an initial hyperactive response during the first 10 minutes, followed immediately (20-50 minutes) by a significant decline in locomotion, relative to the other two groups. Analysis of the visual behavioral ratings taken at 30 minutes following the $\mathrm{AMPH}$ injection showed that the behavioral response to AMPH was qualitatively different in the three groups of animals $\left(\chi^{2}\right.$ statistic $\left.=48.526, p<0.0001\right)$. Whereas $58.8 \%$ of AMPH-pretreated animals withdrawn for 31 days displayed continuous stereotypy (i.e., a rating of 3 or 4), only $10.2 \%$ of AMPH-pretreated animals withdrawn for 2 days and $6.4 \%$ of saline-pretreated controls displayed continuous stereotyped behavior. In contrast,

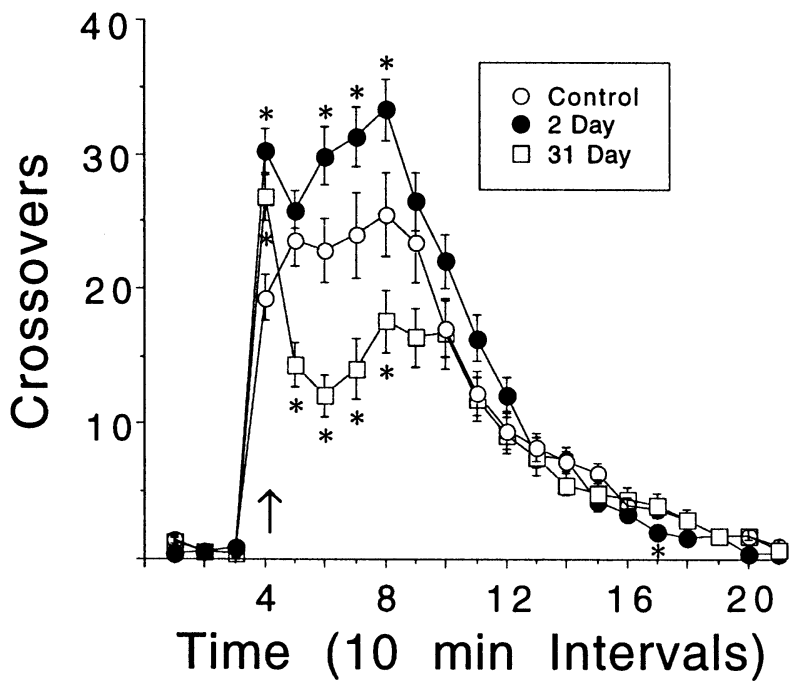

Figure 3. The mean ( \pm S.E.M.) number of crossovers per 10 minute interval in Control animals and animals tested after 2 days or 31 days of withdrawal from AMPH. The first three intervals represent baseline activity. The arrow represents the time at which animals received a challenge injection of $2.0 \mathrm{mg} / \mathrm{kg}$ of AMPH. The overall ANOVA showed significant group differences in the response to $\mathrm{AMPH}$ (group effect $F=5.12, p<0.007$, interaction effect $F=6.83, p<$ 0.0001). Subsequent ANOVA's comparing two groups at a time revealed that the 2 Day group differed from both Control animals (group effect $F=2.94, p=0.089$, interaction effect $F=4.56, p<0.0001$ ) and the 31 Day group (group effect $F=11.64, p=0.0009$; interaction effect $F=12.56, p<$ $0.0001)$. In addition, the 31 Day group differed from the Control group (group effect $F=2.39, p=0.126$, interaction effect $F=4.74, p<0.0001$ ). The asterisks indicate the time intervals when the 2 Day and 31 Day groups differed from Controls. Group $n$ values: Control $n=56$; 2 Day $n=67 ; 31$ Day $n=32$. 
$76.3 \%$ of the animals withdrawn from AMPH for 2 days displayed hyperlocomotion, whereas $38.2 \%$ of AMPHpretreated animals withdrawn for 31 days and $59.6 \%$ of saline-pretreated controls displayed hyperlocomotion at 30 minutes following the AMPH injection.

\section{Microdialysis}

Basal Values. Figure 4 shows the basal concentrations of NE in the hypothalamus and hippocampus for each of the three groups during both the first and second dialysis test sessions. In the hypothalamus, there were significant group differences during the first dialysis session, but not during the second test session. Animals withdrawn from AMPH for one day had significantly higher basal NE levels than both control animals and animals withdrawn from AMPH for 30 days. These latter two groups did not differ from each other. In the hippocampus, basal dialysate NE concentrations were significantly elevated in animals tested after either 1 or 30 days of withdrawal, relative to controls, and this was evident during both dialysis test sessions.

Stimulated Values. Figure 5 shows the effects of a challenge injection of AMPH on the concentration of NE in dialysate obtained from either the hypothalamus or hippocampus during the second dialysis test session. AMPH produced a significant increase in NE in both brain regions, as expected (Kuczenski and Segal 1992), but there were also regionally specific group differences in the magnitude of the increase in NE, which varied as a function of how the data were expressed.

In the hypothalamus, there were no significant group differences when the data were expressed in $\mathrm{pg} / \mu \mathrm{l}$ (Figure 5A). As there were no group differences in basal NE in the hypothalamus, however, the stimulated values were also expressed as a percent of the average basal value (first three intervals) for each animal to control for individual variation in dialysate NE concentrations. These data are shown in Figure 5C. When expressed in this way there were significant group differences in the response to an AMPH challenge. AMPH pretreated animals tested after two days of withdrawal showed a significantly smaller response than control animals, whereas AMPH pretreated animals tested after 31 days of withdrawal showed a significantly augmented response (Figure 5C).

In the hippocampus there were significant group differences in the concentration of NE in dialysate $(\mathrm{pg} / \mu \mathrm{l})$ following the AMPH challenge, as shown in Fig. 5B. Following the AMPH challenge the concentration of NE was significantly higher in animals tested 31 days after the discontinuation of AMPH treatment than in control animals. Although there was a similar trend in animals tested after 2 days of withdrawal it was not quite statistically significant. However, the effect of AMPH pre-

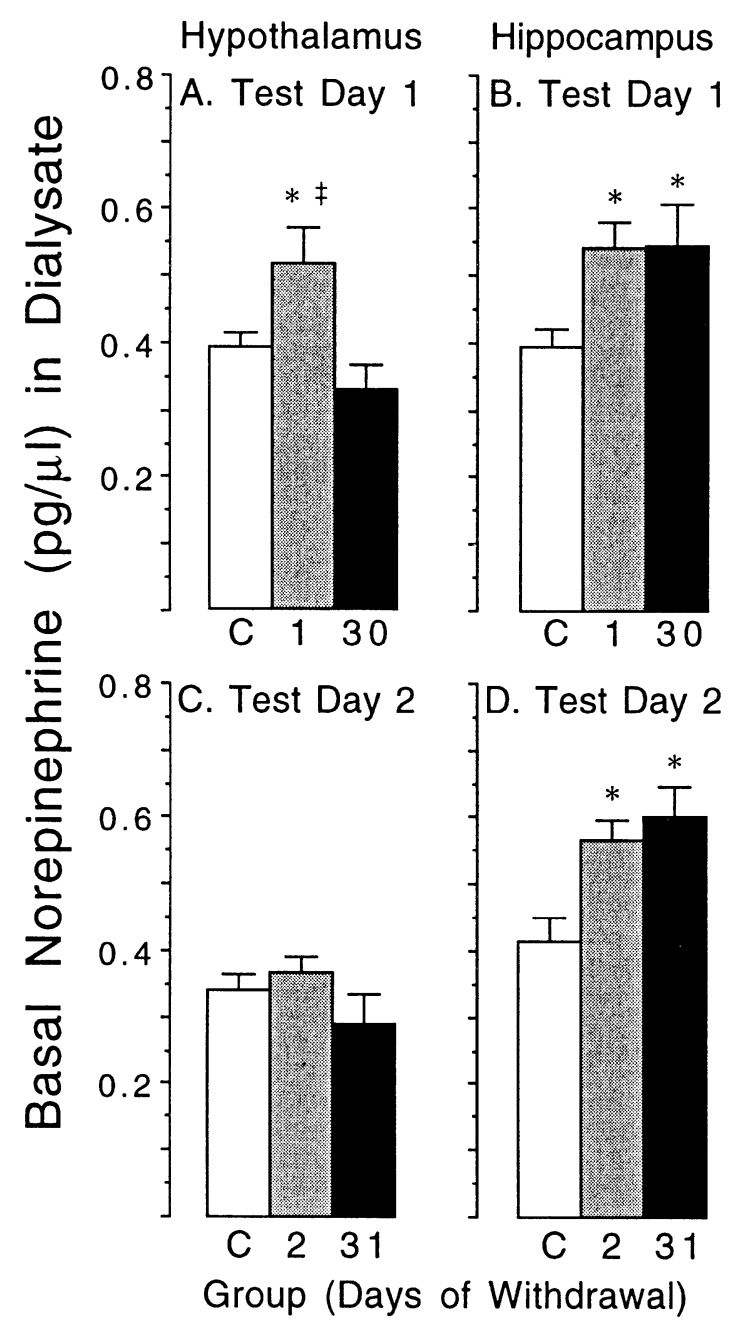

Figure 4. The mean (+S.E.M.) basal concentration of NE in dialysate obtained from the hypothalamus or hippocampus of control animals (C) and animals tested after both 1 and 2 days of AMPH withdrawal, or after 30 and 31 days of AMPH withdrawal. Panels A and B represent values obtained during the first dialysis test day, and Panels $C$ and $D$ represent values obtained 24 hours later in the same animals. There was a significant effect of AMPH withdrawal on dialysate NE in the hypothalamus on the first dialysis test day $(F=6.30, p=0.004)$, but not on the second test day $(F=$ $1.53, p=0.23)$. In the hippocampus, there was a significant effect of AMPH withdrawal on dialysate NE on both the first $(F=3.97, p=0.027)$ and the second dialysis test days $(F=$ $7.81, p=0.0012)$. The asterisks represent a difference from Control animals $(p<0.05$, as indicated by Fisher's PLSD tests), and the double cross represents a difference from the 30 Day group $(p<0.05)$. Group $n$ values: Hypothalamus control, $n=17-21$; hypothalamus 1-2 Day, $n=14-16$; hypothalamus 30-31 Day, $n=9-13$; hippocampus control, $n=$ 14-16; hippocampus 1-2 Day, $n=17-20$; hippocampus 3031 Day, $n=14-16$. Group $n$ values vary because data were not available for every animal during both dialysis test days. 
Hypothalamus
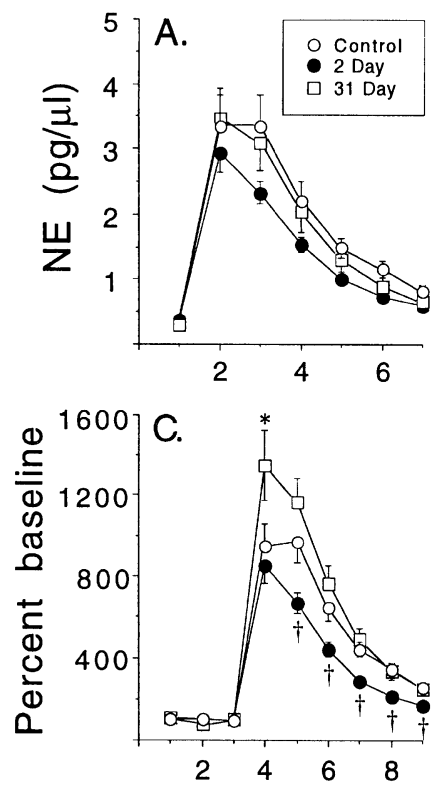

Time
B.

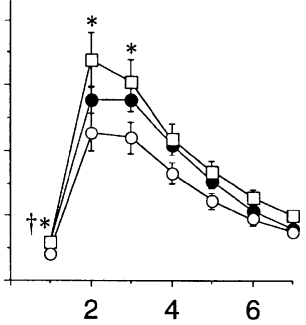

D.

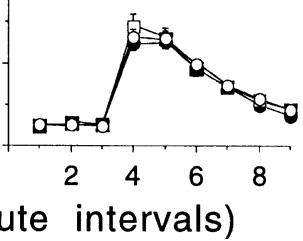

Hippocampus

Figure 5. The mean ( \pm S.E.M.) concentration of NE in 30 minute dialysis samples obtained from the hypothalamus or hippocampus in Control animals and animals tested after 2 days or 31 days of AMPH withdrawal. Panels A and B represent the raw data (in pg/ $\mu \mathrm{l}$ ), and Panels $C$ and $D$ represent the same data expressed as a percent of each animal's baseline. Animals were given an injection of $2.0 \mathrm{mg} / \mathrm{kg}$ of AMPH at the time indicated by the arrow. ANOVA's were performed on the stimulated NE values only (i.e., the basal values were excluded). There was a significant effect of AMPH withdrawal on the NE response to an AMPH challenge in the hypothalamus when the data were expressed as a percent of baseline (group effect $F=6.61, p=0.004$; interaction effect $F=4.45, p<0.0001)$, but not when the raw data were analyzed (group effect $F=1.69, p=0.20$; interaction effect $F=0.72, p=0.70)$. Subsequent ANOVA's performed on the percent of baseline data comparing two groups at a time revealed that animals tested after 2 days of AMPH withdrawal differed significantly from both Controls (group effect $F=6.65, p=0.016$; interaction effect $F=2.87, p=$ 0.005 ) and the 31 Day group (group effect $F=15.09, p=$ 0.0009 ; interaction effect $F=9.33, p<0.0001$ ). In addition, the 31 Day group differed significantly from Control animals (group effect $F=2.01, p=0.17$; interaction effect $F=3.11$, $p=0.0025)$. In the hippocampus, there were significant group differences in the effect of AMPH withdrawal on the $\mathrm{NE}$ response to an $\mathrm{AMPH}$ challenge when the raw data were analyzed (group effect $F=2.99, p=0.060$; interaction effect $F=2.39, p=0.010)$, but not when the data were expressed as a percent of baseline $(F<0.5)$. Subsequent ANOVA's performed on the raw data comparing two groups at a time revealed that both AMPH-pretreated groups differed significantly from Control animals (2 Day versus Control: group effect $F=2.74, p=0.11$; interaction effect $F=2.52, p=$ 0.032; 31 Day versus Control: group effect $F=5.02, p=$ 0.034 ; interaction effect $F=3.48, p=0.006$ ). The crosses represent a difference between 2 Day and Control animals $p<$ treatment on the response to an AMPH challenge was proportional to what would be expected by group differences in the basal concentrations of NE described above (Figure 4B \& D). If the group differences in basal NE were eliminated by expressing the data as a percent of baseline the group differences in the response to the AMPH challenge were eliminated, as illustrated in Figure 5D.

\section{DISCUSSION}

Three major effects of repeated AMPH treatment on brain and behavior are reported here. First, repeated exposure to AMPH resulted in time-dependent changes in both spontaneous locomotor activity and in the psychomotor response to a subsequent challenge injection of AMPH. Second, repeated exposure to AMPH resulted in time-dependent and regionally-specific changes in the basal concentrations of NE in dialysate. Third, repeated exposure to AMPH resulted in time-dependent and regionally-specific changes in the NE response to an AMPH challenge.

When tested 1-2 days following the discontinuation of repeated treatment with AMPH rats showed a significant depression in spontaneous nocturnal locomotor activity, but this dissipated within 30 days. This is consistent with a number of reports of post-AMPH withdrawal behavioral depression lasting for a few days to a week, depending on the AMPH treatment regimen (Paulson et al. 1991; Robinson and Camp 1987; Segal and Mandell 1974). Behavioral depression is one of a number of symptoms that has been associated with psychostimulant withdrawal syndromes (Kokkinidis 1988; Markou and Koob 1991). Others include a number of changes in motivated behavior (Carroll and Lac 1987; Cassens et al. 1981; Kokkinidis et al. 1986; Kokkinidis and Zacharko 1980; Leith and Barrett 1976; Schreiber et al. 1976).

The AMPH withdrawal syndrome is presumably due to transient drug-induced adaptations in neural systems involved in psychomotor activation and reward, but its neural basis is not well understood. Much of the focus in this area has been on withdrawal-related adaptations in DA systems and how these might contribute to changes in motivated behavior (Barrett and White 1980; Crippens et al. 1993; Kokkinidis 1988; Paulson and Robinson 1995, 1996; Rossetti et al. 1992). The

0.05, as indicated by Fisher's PLSD tests), and the asterisks represent a difference between 31 Day and Control animals $(p<0.05)$. Group $n$ values: Hypothalamus Control, $n=17$; hypothalamus 2 Day, $n=13$; hypothalamus 31 Day, $n=9$; hippocampus Control, $n=16$; hippocampus 2 Day, $n=20$; hippocampus 31 Day, $n=12$. 
present study suggests, however, that repeated AMPH treatment also produces adaptations in NE systems. The basal concentration of NE in dialysate was elevated in both the hypothalamus and hippocampus 1 day after the discontinuation of AMPH treatment, and remained elevated in the hippocampus even after 30 days of withdrawal. Although it is not clear how these changes in NE neurotransmission contribute to the AMPH withdrawal syndrome, they are consistent with the idea that AMPH exposure can produce alterations in NE systems that outlast the acute effects of the drug (Alloway and Rebec 1983; Bardsley and Bachelard 1981; Cassens et al. 1979; Eichler et al. 1980; Herman et al. 1971; Tonge 1974; Short and Shuster 1976; Lynch et al. 1977; Pearl and Seiden 1976; Segal et al. 1980; Sorenson et al. 1985). We previously reported, for example, that AMPH withdrawal is accompanied by a transient decrease in hypothalamic NE content (Paulson et al. 1991), and the present study suggests this could be due to an increase in basal NE release.

In the present study there were regional differences in the effects of AMPH pretreatment on basal NE concentrations. This suggests that chronic exposure to AMPH may have different effects on different NE cell populations. In the hypothalamus there was only a very transient change in basal NE concentrations. The NE innervation of the paraventricular region of the hypothalamus arises from a number of sources, but most of the input is from ventrally-located NE cell groups in the midbrain (Lindvall and Bjorkland 1983; Moore and Bloom 1979). In the hippocampus, however, there was a long-lasting (at least 30 days) elevation in basal NE. The NE innervation of the hippocampus arises almost exclusively from the locus coeruleus (Loy et al. 1980). Therefore, AMPH may produce more long-lasting tonic adaptations in dorsally-located NE cell populations than in ventrally-located $\mathrm{NE}$ cell groups.

It is worth noting that an increase in basal NE neurotransmission, similar to that reported here, has also been associated with the behavioral depression that occurs following uncontrollable stress (Weiss 1991), as well as following withdrawal from opiates and benzodiazepines (Grant et al. 1985; Redmond and Huang 1982; Redmond and Krystal 1984). For example, the stress-induced depression produced by exposure to uncontrollable stress is associated with a transient depletion of NE content in the hypothalamus and the locus coeruleus, and like that of opiate withdrawal, is apparently due to increased NE release and turnover in NE terminal regions (Weiss et al. 1980; Weiss and Simson 1986; see Zacharko and Anisman 1989). The notion that similar changes in NE neurotransmission are at least partially responsible for both the AMPH withdrawal syndrome and the consequences of uncontrollable stress is supported by reports that tricyclic antidepressants alleviate the symptoms of both syndromes (Lynch and
Leonard 1978; Seltzer and Tonge 1975; Zacharko et al. 1984; Zacharko and Anisman 1989).

Prior treatment with AMPH not only altered spontaneous motor behavior and the basal levels of NE in dialysate, but also the response to a subsequent challenge injection of AMPH. Animals given an AMPH challenge 2 days after the discontinuation of AMPH pretreatment showed a small increase in locomotor activity, relative to saline pretreated control animals, indicative of sensitization. When given an AMPH challenge after 31 days of withdrawal, however, AMPH pretreated animals showed a significant decrease in their locomotor response, relative to control animals, which was due to the emergence of focussed stereotyped behaviors. Focussed stereotyped behavior is characteristic of higher doses of AMPH than locomotor activity (Segal and Mandell 1974), suggesting that animals expressed greater sensitization when tested 31 days after the discontinuation of AMPH pretreatment than when tested after only 2 days. This time-dependent emergence of behavioral sensitization has been described many times before (Hitzemann et al. 1977; Kolta et al. 1985; see Antelman 1988 for review), especially after pretreatment with escalating doses of AMPH (Paulson et al. 1991).

There were also time and regionally-dependent effects on the NE response to an AMPH challenge, although it is not clear how or whether these contribute to the expression of behavioral sensitization. One of the most interesting findings was that $\mathrm{AMPH}$ pretreatment resulted in a very persistent increase in the amount of NE in dialysate obtained from the hippocampus, both before and after an AMPH challenge. This effect is quite different from that described in DA systems. Animals sensitized to AMPH and then tested after weeks to months of withdrawal show normal basal levels of DA in dialysate from the nucleus accumbens or caudate (Crippens et al. 1993; Paulson and Robinson 1995, 1996; Robinson et al. 1988). They often show, however, an increased DA response to an AMPH challenge (Kazahaya et al. 1989; Patrick et al. 1991; Paulson and Robinson 1995; Robinson et al. 1988; Vezina 1993; Wolf et al. 1993). This increased responsivity to an AMPH challenge is typically evident early after the discontinuation of AMPH treatment, but may not be evident early after the discontinuation of AMPH treatment (Paulson and Robinson 1995; Segal and Kuczenski 1992; Wolf et al. 1993). Thus, in DA systems the effect of AMPH pretreatment seems to be to enhance responsiveness to a subsequent challenge with AMPH. In the present case AMPH pretreatment elevated the concentration of NE in hippocampal dialysate following an AMPH challenge, but the magnitude of this effect was exactly what would be predicted by the magnitude of the increase in basal NE. Thus, it seems that in the hippocampus (and perhaps in the entire locus coeruleus projection field) AMPH pretreatment may produce a kind of persistent, 
"tonic" elevation in NE neurotransmission, and not specifically an increase in responsiveness to an AMPH challenge.

The effect of AMPH pretreatment on the NE response in the hypothalamus was quite different from that in the hippocampus. Indeed, the hypothalamic NE response to an AMPH challenge is difficult to interpret because, like the hippocampal NE response, the effect varied as a function of how the data were expressed. When the raw data $(\mathrm{pg} / \mu \mathrm{l})$ were used there was no significant effect of AMPH pretreatment on the concentration of NE in hypothalamic dialysate following an AMPH challenge (Figure 5A). When stimulated NE values were expressed as a percent of the baseline, however, there were statistically significant group differences in the NE response to an AMPH challenge. The NE response was significantly depressed in AMPH pretreated animals tested after only 2 days of withdrawal, but was significantly enhanced in animals tested after 31 days of withdrawal (Fig. 5C). There were no group differences in basal NE levels in animals tested either 2 or 31 days after withdrawal. Thus, to the extent that the response to AMPH, relative to the basal level of NE, reflects a drug-induced adaptation in the responsiveness of hypothalamic NE systems to a challenge, there are quite different adaptations expressed early versus late after the discontinuation of AMPH treatment. This kind of time-dependent variation in neurotransmission as a function of time after the discontinuation of psychostimulant treatment is consistent with many other observations (Antelman 1988; Kalivas and Duffy 1993; Paulson and Robinson 1995, 1996; Rosetti et al. 1992; Wolf et al. 1993). The suggestion that AMPH pretreatment may result in a persistent increase in the responsiveness of NE neurons is also consistent with previous electrophysiological studies (Harris and Williams 1992; Sorensen et al. 1982), and reports that prior exposure to stress enhances NE neurotransmission in response to subsequent stress (Abercrombie et al. 1988; Nisenbaum et al. 1991; see Glavin 1985; Zacharko and Anisman 1989 for reviews).

It is difficult to say with any certainty, however, whether stimulated dialysate values are better expressed as a percent of basal values or as the concentration of transmitter quantified in dialysate. This question has received relatively little attention in the microdialysis literature. Indeed, these two sets of data may reflect different processes; i.e., the absolute amount of transmitter available to interact with a receptor versus the change in the amount of transmitter at a receptor relative to the resting state. Which is more relevant: the absolute amount or the magnitude of the change? Of course, if the same pattern of results are obtained regardless of how the data are expressed this is a moot point. But if the results vary as a function of how the data are expressed, as is the case here, and the reader is provided only with the data expressed as a percent of baseline (which is usually the case), the reader is not allowed the opportunity to interpret the results. When there is a discrepancy it would seem that the prudent course would be to show both sets of data, and then, as we learn more about this methodology, data can be subject to reinterpretation at a later date. Data that are not provided are lost for this purpose.

In summary, the data presented here indicate that repeated exposure to AMPH produces time-dependent and regionally-specific adaptations in NE systems projecting to the hippocampus and hypothalamus. Some of these adaptations persist for a very long period of time (up to a month) following the discontinuation of AMPH treatment. AMPH-induced adaptations in NE neurotransmission may contribute, therefore, to both the AMPH withdrawal syndrome and to the expression of behavioral sensitization, along with drug-induced adaptations in other catecholamine systems.

\section{ACKNOWLEDGMENTS}

This research was supported by grant 04294 from the National Institute on Drug Abuse to Terry Robinson. We would like to thank Buda Martonyi for his technical assistance and Gianh Nguyen for her help in conducting this experiment.

\section{REFERENCES}

Abercrombie ED, Keller RW, Zigmond MJ (1988): Characterization of hippocampal norepinephrine release as measured by microdialysis perfusion: Pharmacological and behavioral studies. Neuroscience 27:897-904

Abercrombie E, Finley J (1991): Monitoring extracellular norepinephrine in brain using in vivo microdialysis and HPLC-EC. In Robinson TE, Justice Jr JB (eds), Microdialysis in the Neurosciences, Amsterdam, Elsevier, pp 253-274

Alloway KD, Rebec GV (1983): Shift from inhibition to excitation in the neostriatum but not in the nucleus accumbens following long-term amphetamine. Brain Res 273: 71-79

Antelman S (1988): Time-dependent sensitization as the cornerstone for a new approach to pharmacotherapy: Drugs as foreign/stressful stimuli. Drug Dev Res 14:1-30

Barrett RS, White DK (1980): Reward system depression following chronic amphetamine: Antagonism by haloperidol. Pharmacol Biochem Behav 13:555-559

Bardsley ME, Bachelard HS (1981): Catecholamine levels and tyrosine hydroxylase activities in rat brain regions after chronic treatment with, and withdrawal of methamphetamine. Biochem Pharmacol 30:1543-1549

Carroll ME, Lac ST (1987): Cocaine withdrawal produces behavioral disruptions in rats. Life Sci 40:2183-2190

Cassens G, Actor C, Kling M, Schildkraut JJ (1981): Amphetamine withdrawal: Effects on threshold of intracranial reinforcement. Psychopharmacology 73:318-322 
Cassens G, Kuruc A, Orsulak PJ, Schildkraut JJ (1979): Amphetamine withdrawal: Effects on brain levels of MHPG-SO ${ }_{4}$ in the rat. Commun Psychopharmacol $3: 217-223$

Crippens D, Camp DM, Robinson TE (1993): Basal extracellular dopamine in the nucleus accumbens during amphetamine withdrawal: "No net flux" microdialysis study. Neurosci Lett 164:145-148

Eichler AJ, Antelman SM, Black CA (1980): Amphetamine stereotypy is not a homogeneous phenomenon: Sniffing and licking show distinct profiles of sensitization and tolerance. Psychopharmacology 68:287-290

Gawin FH, Ellinwood EJ (1988): Cocaine and other stimulants. Actions, abuse, and treatment. N Engl J Med 318: 1173-1182

Glavin GB (1985): Stress and brain noradrenaline: A review. Neurosci Biobehav Rev 9:233-243

Grant SJ, Galloway MP, Mayor R, Fenerty JP, Finkelstein MF, Roth RH, Redmond DJ (1985): Precipitated diazepam withdrawal elevates noradrenergic metabolism in primate brain. Eur J Pharmacol 107:127-132

Harris GC, Williams JT (1992): Sensitization of locus ceruleus neurons during withdrawal from chronic stimulants and antidepressants. J Pharmacol Exp Ther 261:476-483

Herman ZS, Trzeciak H, Chrusciel TL, Kmieciak KK, Drybanski A, Sokola A (1971): The influence of prolonged amphetamine treatment and amphetamine withdrawal on brain biogenic amine content and behaviour in the rat. Psychopharmacologia 21:74-81

Hitzemann RJ, Tseng LF, Hitzemann BA, Sampath-Khanna S, Loh HH (1977): Effects of withdrawal from chronic amphetamine intoxication on exploratory and stereotyped behaviors in the rat. Psychopharmacology 54:295-302

Jaffe JH (1990): Drug addiction and drug abuse. In Gilman AG, Rall TW, Nies AS, Taylor P (eds), The Pharmacological Basis of Therapeutics. New York, Pegamon Press, pp 522-573

Kalivas PW, Duffy P (1993): Time course of extracellular dopamine and behavioral sensitization to cocaine: I. Dopamine axon terminals. J Neurosci 13:266-275

Kalivas PW, Stewart J (1991): Dopamine transmission in the initiation and expression of drug- and stress-induced sensitization of motor activity. Brain Res Rev 16:223-244

Kazahaya Y, Akimoto K, Otsuki S (1989): Subchronic methamphetamine treatment enhances methamphetamineor cocaine-induced dopamine efflux in vivo. Biol Psychiatry 25:903-912

Kokkinidis L (1988): Neurochemical correlates of postamphetamine depression and sensitization in animals. Anim Models Psychiatr Disord 2:148-173

Kokkinidis L, Zacharko RM (1980): Response sensitization and depression following long-term amphetamine treatment in a self-stimulation paradigm. Psychopharmacology 68:73-76

Kokkinidis L, Zacharko RM, Anisman H (1986): Amphetamine withdrawal: A behavioral evaluation. Life Sci 38: 1617-1623

Kolta MG, Shreve P, De Souza V, Uretsky NJ (1985): Time course of the development of the enhanced behavioral and biochemical responses to amphetamine after pre- treatment with amphetamine. Neuropharmacology 24: 823-829

Kuczenski R, Segal DS (1992): Regional norepinephrine response to amphetamine using dialysis: Comparison with caudate dopamine. Synapse 11:164-169

Leith NJ, Barrett RJ (1976): Amphetamine and the reward system: Evidence for tolerance and post-drug depression. Psychopharmacologia 46:19-25

Lett BT (1989): Repeated exposures intensify rather than diminish the rewarding effects of amphetamine, morphine, and cocaine. Psychopharmacology (Berlin) 98: 357-362

L'Heureux RL, Dennis T, Curet O, Scatton B (1986): Measurement of endogenous noradrenaline release in the rat cerebral cortex in vivo by transcortical dialysis: Effects of drugs affecting noradrenergic transmission. J Neurochem 46:1794-1801

Lindvall O, Bjorklund A (1983): Dopamine- and norepinephrine-containing neuron systems: Their anatomy in the rat brain. In Emson PC (ed), Chemical Neuroanatomy. New York, Raven Press, pp 229-255

Loy R, Koziell DA, Lindsey JD, Moore RY (1980): Noradrenergic innervation of the adult rat hippocampal formation. J Comp Neurol 189:699-710

Lynch M, Kenny M, Leonard BE (1977): The effect of chronic administration of $d$-amphetamine on regional changes in catecholamines in the rat brain. J Neurosci Res 3:295-300

Lynch MA, Leonard BE (1978): Changes in brain gammaaminobutyric acid concentrations following acute and chronic amphetamine administration and during post amphetamine depression. Biochem Pharmacol 27:18531855

Markou A, Koob GF (1991): Postcocaine anhedonia: An animal model of cocaine withdrawal. Neuropsychopharmacology 4:17-26

Moore RY, Bloom FE (1979): Central catecholamine neuron systems: Anatomy and physiology of the norepinephrine and epinephrine systems. Ann Rev Neurosci 2:113-168

Nisenbaum LK, Zigmond MJ, Sved AF, Abercrombie ED (1991): Prior exposure to chronic stress results in enhanced synthesis and release of hippocampal norepinephrine in response to a novel stressor. J Neurosci 11:1478-1484

Patrick SL, Thompson TL, Walker JM, Patrick RL (1991): Concomitant sensitization of amphetamine-induced behavioral stimulation and in vivo dopamine release from the rat caudate nucleus. Brain Res 538:343-346

Paulson PE, Camp DM, Robinson TE (1991): Time course of transient behavioral depression and persistent behavioral sensitization in relation to regional brain monoamine concentrations during amphetamine withdrawal in rats. Psychopharmacology 103:480-492

Paulson PE, Robinson PE (1995): Amphetamine-induced time-dependent sensitization of dopamine neurotransmission in the dorsal and ventral striatum: A microdialysis study in behaving rats. Synapse 19:56-65

Paulson PE, Robinson TE (1996): Regional differences in the effects of amphetamine withdrawal on dopamine dynamics in the striatum: Analysis of circadian patterns using automated on-line microdialysis. Neuropsychopharmacology 14:325-337 
Paxinos G, Watson C (1986): The Rat Brain in Stereotaxic Coordinates. New York, Raven Press

Pearl RG, Seiden LS (1976): The existence of tolerance to and cross-tolerance between $d$-amphetamine administration and methylphenidate for their effects on milk consumption and on differential reinforcement of low rate performance in the rat. J Pharmacol Exp Ther 198:635-647

Piazza PV, Deminiere JM, Le MM, Simon H (1989): Factors that predict individual vulnerability to amphetamine self-administration. Science 245:1511-1513

Redmond DE, Huang YH (1982): The primate locus coeruleus and effects of clonidine on opiate withdrawal. J Clin Psychiat 43 (Suppl 6):25-29

Redmond DE, Krystal JH (1984): Multiple mechanisms of withdrawal from opioid drugs. Ann Rev Neurosci 7: $443-478$

Robinson TE, Becker JB (1986): Enduring changes in brain and behavior produced by chronic amphetamine administration: A review and evaluation of animal models of amphetamine psychosis. Brain Res Rev 396: 157-198

Robinson TE, Camp DM (1987): Long-lasting effects of escalating doses of $d$-amphetamine on brain monoamines, amphetamine-induced stereotyped behavior and spontaneous nocturnal locomotion. Pharmacol Biochem Behav 26:821-827

Robinson TE, Camp DM (1991): The feasibility of repeated microdialysis for within-subjects design experiments: Studies on the mesostriatal dopamine system. In Robinson TE, Justice Jr JB (eds), Microdialysis in the Neurosciences. Amsterdam, Elsevier, pp 189-234

Robinson TE, Jurson PA, Bennett JA, Bentgen KM (1988): Persistent sensitization of dopamine neurotransmission in ventral striatum (nucleus accumbens) produced by past experience with (+)-amphetamine: A microdialysis study in freely moving rats. Brain Res 462:211-222

Rossetti ZL, Hmaidan Y, Gessa GL (1992): Marked inhibition of mesolimbic dopamine release: A common feature of ethanol, morphine, cocaine and amphetamine abstinence in rats. Eur J Pharmacol 221:227-234

Sato M, Chen CC, Akiyama K, Otsuki S (1983): Acute exacerbation of paranoid psychotic state after long-term abstinence in patients with previous methamphetamine psychosis. Biol Psychiatry 18:429-440

Schreiber H, Bell R, Conely L, Kufner M, Palet J, Wright L (1976): Diminished reaction to a novel stimulus during amphetamine withdrawal in rats. Pharmacol Biochem Behav 5:687-690

Segal DS, Kuczenski R (1992): In vivo microdialysis reveals a diminished amphetamine-induced DA response corresponding to behavioral sensitization produced by repeated amphetamine pretreatment. Brain Res 571: 330-337

Segal DS, Mandell AJ (1974): Long-term administration of $d-$ amphetamine: Progressive augmentation of motor activity and stereotypy. Pharmacol Biochem Behav 2:249-255

Segal DS, Weinberger SB, Cahill J, McCunney SJ (1980): Multiple daily amphetamine administration: Behavioral and neurochemical alterations. Science 207:904-907
Seltzer V, Tonge SR (1975): Methylamphetamine withdrawal as a model for the depressive state: Antagonism of postamphetamine depression by imipramine. J Pharm Pharmacol 27:16P

Short PH, Shuster L (1976): Changes in brain norepinephrine associated with sensitization to $d$-amphetamine. Psychopharmacology 48:59-67

Sorensen SM, Hattox S, Johnson SW, Bickford P, Murphy R, Freedman R (1985): Norepinephrine-dependent and independent mechanisms of persistent effects of amphetamine in rat cerebellum. Life Sci 36:2383-2389

Sorensen SM, Johnson SW, Freedman R (1982): Persistent effects of amphetamine on cerebellar purkinje neurons following chronic administration. Brain Res 247:365-371

Stewart J, Badiani A (1993): Tolerance and sensitization to the behavioral effects of drugs. Behav Pharmacol 4:289-312

Tonge SR (1974): Noradrenaline and 5-hydroxytryptamine metabolism in six areas of rat brain during post-amphetamine depression. Psychopharmacologia 38:181-186

Valadez A, Schenk S (1994): Persistence of the ability of amphetamine preexposure to facilitate acquisition of cocaine self-administration. Pharmacol Biochem Behav 47:203-205

Vezina P (1993): Amphetamine injected into the ventral tegmental area sensitizes the nucleus accumbens dopaminergic response to systemic amphetamine: An in vivo microdialysis study in the rat. Brain Res 605:332-337

Watson R, Hartmann E, Schildkraut JJ (1972): Amphetamine withdrawal: Affective state, sleep patterns, and MHPG excretion. Am J Psychiatry 129:263-269

Weiss JM (1991): Stress-induced depression: Critical neurochemical and electrophysiological changes. In Madden J (ed), Neurobiology of Learning, Emotion and Affect. New York, Raven Press, pp 123-154

Weiss JM, Bailey WH, Pohorecky LA, Korzeniowski D, Grillione G (1980): Stress-induced depression of motor activity correlates with regional changes in brain norepinephrine but not in dopamine. Neurochem Res 5:9-22

Weiss JM, Simson PG (1986): Depression in an animal model: Focus on the locus ceruleus. In Murphy D (ed), Antidepressants and Receptor Function (Ciba Foundation Symposium 123). Chichester, John Wiley, pp 191-215

White FJ, Wolf ME (1991): Psychomotor stimulants. In Pratt J (ed), The Biological Bases of Drug Tolerance and Dependence. New York, Academic Press, pp 153-197

Wolf ME, White FJ, Nassar R, Brooderson RJ, Khansa MR (1993): Differential development of autoreceptor subsensitivity and enhanced dopamine release during amphetamine sensitization. J Pharmacol Exp Ther 264: 249-255

Zacharko RM, Anisman H (1989): Pharmacological, biochemical, and behavioral analyses of depression: Animal models. In Koob G (ed), Animal Models of Depression. Boston, Birkhauser, pp 204-238

Zacharko RM, Bowers WJ, Kelley MS, Anisman H (1984): Prevention of stressor-induced disturbances of self-stimulation by desmethylimipramine. Brain Res 321:175-179 